\title{
Enseñar filosofía: el cómo es el qué
}

\author{
Por Enver J. Torregroza L. \\ Filósofo, Universidad Nacional de Colombia, \\ Magíster en Filosofía, Pontificia Universidad Javeriana \\ Profesor Principal \\ Universidad del Rosario.
} Facultades de Ciencia Política y Gobierno y de Relaciones Internacionales

Resumen: El propósito de este artículo es enunciar de forma general cuál puede ser la utilidad de la enseñanza de la filosofía en el bachillerato, explicada -por pura comodidad- en el lenguaje contemporáneo de las "competencias". Se defiende la idea de que la enseñanza de la filosofía presupone una cierta manera de entender la naturaleza de la actividad filosófica según la cual enseñar a filosofar, y no enseñar a hablar doctrinariamente o a ser "críticos de la realidad social" o cosas por el estilo, resulta esencial no sólo para el educando, sino también para la filosofía misma.

Palabras clave: enseñanza, filosofía, bachillerato, competencias, lógica, ética.

\begin{abstract}
The article aims to show generally the benefits of philosophy teaching in high schools, and, as is usual, explained it in terms of "competences". A particular way to understanding the philosophy practice is supposed in the philosophy teaching. According to it "teach to do philosophy" rather than "teach to speak in doctrinal language" or than "teach to criticizing the social reality" and so on, becomes essential to the student and to philosophy itself.
\end{abstract}

Key words: teaching, philosophy, high school, competence, logic, ethics. 


\section{Introducción}

$\mathrm{E}$

1 problema fundamental con el que se enfrenta todo profesor de filosofía es el de establecer claramente qué y cómo enseñar. Esta dificultad no puede, en el caso de la filosofía, obtener una respuesta trivial, debido al carácter problemático de la actividad filosófica. ¿Se puede enseñar la filosofía? ¿Existe algo así como "la filosofía" que pueda ser enseñado? La filosofía evidentemente no se comporta como cualquier otra materia del conocimiento humano. No todos están de acuerdo sobre qué tipo de conocimiento es. Es más, su condición de conocimiento no está completamente clara. Lo que sí sabemos con certeza es que la filosofía no es una: se trata de una actividad, variada y diversa. Esto no significa que no se puedan encontrar puntos en común entre las filosofías de todos los tiempos. Si esto no fuese así, no sería posible que habláramos en general de filosofía. Existen entre las filosofías de todas las épocas ciertos parecidos de familia. Pero esos parecidos de familia sólo se pueden establecer haciendo filosofía, reflexionando sobre la materia de manera comprensiva, e interpretando desde nuestra situación histórica la historia misma de la labor filosófica. En resumidas cuentas no se puede enseñar filosofía sin hacer filosofía.

Un problema adicional surge cuando se quiere pensar en la enseñanza de la filosofía en el bachillerato. Cuando la filosofía se la enseña a los que quieren estudiarla se dan por sentadas muchas cosas. Pero cuando la enseñanza está dirigida hacia un grupo de personas que no necesariamente se van a consagrar a la actividad filosófica es necesario pensar para qué les puede servir el estudio de la filosofía. Esto significa que la enseñanza de la filosofía en el bachillerato presupone una actitud filosófica ya definida, al menos en sus líneas generales. Tal actitud filosófica se hace explícita en los principios pedagógicos que el profesor asume conciente $\mathrm{o}$ inconscientemente.

A continuación voy a exponer lo que considero necesario tener en cuenta para la enseñanza de la filosofía dirigida a estudiantes de bachillerato. Esta propuesta puede servir simplemente de punto de partida para una discusión y no necesariamente está atada a una filosofía particular, aunque un análisis filosófico profundo de la misma podría revelar cierto espíritu filosófico particular latente. El que ese espíritu filosófico propio salga a la luz es necesario, en la medida en que reconozcamos que no hay posturas filosóficas neutras y que después de todo tenemos que tomar partido. Sobre todo, tomar partido por la filosofía. Lo que pienso es que el simple hecho de creer que hay filosofías, que estas se pueden enseñar, que tienen un método y una utilidad, revela ya una postura filosófica lo suficientemente fuerte como para pensar que lo dicho aquí pueda valer en todo contexto. Mi principal interés es hacer evidente que el quehacer pedagógico que comparte esas creencias comparte también una serie características que se pueden definir, al menos, desde una perspectiva general. 


\section{Para qué puede servir el estudio de la filosofía}

El estudio de la filosofía permite el desarrollo de varias competencias ${ }^{1}$ intelectuales, imprescindibles no sólo para las labores académicas en general sino también para el correcto desenvolvimiento del individuo en la vida misma. Tales competencias intelectuales incluyen competencias discursivas, competencias lógicas básicas específicas y competencias intelectuales generales.

Las competencias discursivas tienen que ver con la capacidad de expresarse de manera correcta y eficaz en una lengua natural dada. También, y en un nivel muy básico, se las puede identificar con habilidades de lecto-escritura. Estas habilidades implican un buen desempeño en el uso de la lengua natural, tanto oral como escrita, haciendo una aplicación correcta de los usos y las normas gramaticales de sintaxis, ortografía y ortología, ampliando continuamente el vocabulario y apropiándose de otros recursos más complejos de la lengua. También implican un buen nivel de comprensión de "textos", hablados como escritos; lo que se llama genéricamente comprensión de lectura. Estas competencias no se pueden desarrollar ni entender de manera desligada de las competencias lógicas. Es más, un buen uso del lenguaje o un dominio elevado del mismo reflejan, requieren de y permiten a su vez una potente capacidad de compresión racional del entorno.

Las competencias lógicas básicas específicas son tan importantes como las anteriores. En principio, las competencias lógicas consisten en la capacidad de pensar en términos conceptuales. Son habilidades complejas que conforman una red donde cada uno de los puntos de cruce del tejido, lo mismo que cada uno de los hilos que lo componen, son estrictamente necesarios para el buen desenvolvimiento del todo. Esta comprensión holista de su funcionamiento es indispensable para su desarrollo práctico. Tales competencias se pueden describir en términos de acciones. Esto es así porque desde el punto de vista pedagógico el pensar puede ser mejor comprendido como un actuar. Evidentemente se trata de una reducción teórica bastante fuerte de la actividad del pensamiento. Sin embargo, esto no desvirtúa su eventual confiabilidad y sentido práctico. Basta con que seamos conscientes de que sea una reducción con propósitos prácticos para que no sea una propuesta arbitraria. Además, semejante reducción no está por fuera del contexto filosófico en el que nos encontramos. El pensamiento de nuestra época hace suficiente énfasis en el lenguaje en acción como el lugar natural de la filosofía y desconfía del mentalismo inexpresable. El intento de describir como un saber hacer las competencias lógicas que se pueden enseñar en una clase de filosofía, al menos corresponde con el espíritu -o la ausencia de espíritude nuestra época. No sobra advertir, en virtud del carácter holista de la propuesta, que la siguiente lista de competencias lógicas básicas específicas no pretende ser exhaustiva y que las categorías expuestas no son necesariamente excluyentes:

- Analizar: Consiste en comprender el significado o el uso de un concepto identificando los conceptos contenidos en el mismo o los conceptos relacionados con el mismo, ya sean los limitados por el concepto original o sus limitantes. A este tipo de análisis se le puede

1 Es la palabra que está de moda. Como mi intención es comunicarme, la uso sin reparos. 
denominar análisis conceptual. La acción de analizar también consiste en identificar las partes de una proposición, en el sentido lógico tradicional del término, lo mismo que en identificar tipos de enunciados, ya sea según los esquemas de la lógica de clases o de la lógica proposicional. Este es el análisis de enunciados. Hace parte también de las competencias analíticas el análisis lógico argumentativo. Este consiste en identificar las partes de un argumento, desde la distinción entre premisas y conclusiones hasta la identificación de la estructura del argumento, sea este formal o informal.

Hay otras competencias analíticas que exigen una mirada más global. En primer lugar se encuentra el análisis teórico, que no es otra cosa que la identificación de las partes de una teoría, entendida como un corpus relativamente ordenado de enunciados entrelazados semántica y sintácticamente. En segundo lugar está el análisis de problemas. En este se trata de identificar los sub-problemas y las variables que hacen parte del problema general.

- Sintetizar: Consiste en subsumir unos conceptos en otros conceptos y en subordinar unos enunciados a otros enunciados más generales (sintesis conceptual y sintesis de enunciados). También consiste en reconstruir en un solo argumento general una serie de argumentos encadenados (sintesis de argumentos), lo mismo que en reconstruir en unos pocos enunciados articulados los contenidos desarrollados en una teoría (sintesis de teorías).

- Abstraer: Consiste en desprender los elementos secundarios del contenido primordial de un concepto para contemplarlo "en abstracto". También consiste en formalizar simbólicamente o con un nuevo aparato conceptual un conjunto de conceptos, enunciados, argumentos o teorías. La formalización es abstracción en la medida en que se separan los componentes secundarios o circunstanciales de los básicos o generales.

- Relacionar: Es vincular razonadamente y de manera sistemática un conjunto de conceptos, enunciados, argumentos o teorías, a partir de la identificación de aspectos comunes o vínculos lógicos de variados tipos existentes entre ellos.

- Razonar: Consiste en ser capaz de identificar y aplicar con suficiencia los instrumentos de conexión lógica entre conceptos y enunciados. Es la habilidad de obtener conclusiones a partir de premisas y de identificar o suponer premisas a partir de tesis dadas. El desarrollo de esta competencia requiere del conocimiento de los diversos tipos de razonamiento, sus partes, sus condiciones lógicas y sus leyes.

- Argumentar: Es una forma del razonar que consiste en defender con razones plausibles los propios pensamientos ante un auditorio cualquiera, ya se trate en un diálogo escrito u oral. Exige el conocimiento de las técnicas argumentativas y retóricas.

- Cuestionar: También llamada "criticar", consiste en problematizar las tesis y las teorías propias y ajenas con base en un ejercicio de análisis, comparación y argumentación. Su preámbulo es la acción de preguntar motivada por la curiosidad, pero su desarrollo exige el conocimiento de herramientas lógicas que le permitan al individuo preguntar razonando.

Las competencias intelectuales generales consisten fundamentalmente en comprender e interpretar el entorno. En el terreno de la filosofía, esto es, en el campo de la reflexión teórica y otras actividades afines, estas competencias, que van acompañadas del desarrollo y aplicación de las competencias lógicas básicas específicas y discursivas, constituyen la 
herramienta fundamental de pensamiento. Si la filosofía se define como un conjunto de problemas, más que como un conjunto de doctrinas, la capacidad de comprender e interpretar estos problemas se convierte en la clave para saber filosofía. Lo ideal es que el estudiante llegue, en el ejercicio de estas competencias, a desarrollar una capacidad de comprensión elevada que le permita, no sólo abordar con los elementos que posee los ejes fundamentales de los problemas filosóficos, sino también ser capaz de leer cualquier texto de carácter filosófico o teórico sin más dificultades que las obvias. Adicionalmente, estas competencias, al ser desarrolladas, permiten que el estudiante pueda desenvolverse con mayor presteza en su ambiente, siendo más capaz de afrontar (y en algunos casos dados de resolver) conflictos y situaciones problemáticas. Por esta razón, las competencias intelectuales generales están estrechamente vinculadas con las competencias éticas y estéticas que la filosofía también ayuda a estimular.

Las competencias intelectuales generales que se desarrollan mediante la enseñanza de la filosofía se pueden entender mejor como partes de un proceso. El orden propuesto aquí no es necesariamente lineal, y el ciclo propuesto puede revertirse en la medida en que se puede iniciar en varios puntos del mismo y esto puede exigir un orden inverso. Semejante orden inverso no es un retroceso, en la medida en que todos los puntos de este proceso significan ya un avance frente a una supuesta situación originaria en la que no están presentes. Tales competencias intelectuales generales son:

- Admirarse: Ya Platón y Aristóteles hablaban de la admiración como origen de la filosofía. No se trata sólo de una sensación o un sentimiento de sorpresa. Esto es secundario, en principio, pues semejante sensación no es una competencia sino una disposición que se despierta, similar a la sensibilidad ética o a la sensibilidad estética. Se trata en lo fundamental de la disposición para reconocer una dificultad, una pregunta, un embrollo conceptual; o para cuestionarse y cuestionar el propio entorno. En términos sencillos esta competencia general consiste en saber hacerse preguntas. Su versión específica es la competencia lógica básica que llamamos cuestionar.

- Investigar: Una buena pregunta siempre motiva el deseo de responderla. Pero el sólo deseo no hace la investigación. Si bien responder preguntas de todo tipo requiere usualmente de métodos específicos, que se pueden enseñar como recetas, la acción de investigar es una competencia general que se cultiva. Este es el terreno del "saber hacer" académico, en el que se pueden integrar las otras competencias.

- Explicar: La explicación es síntoma de entendimiento. Ser capaz de explicar algo es ser capaz de entenderlo, y esto se debe buscar con la enseñanza de la filosofía; que el estudiante sea capaz de explicar (entender) la labor filosófica en general y algunos problemas, métodos y argumentos filosóficos. Para explicar hay que relacionar (vincular con criterios lógicos) y, desde una perspectiva más general, esto no es otra cosa que contextualizar; puesto que algo se puede entender mejor si se lo entiende en su contexto.

- Comprender: Esto es lo que se procura con todo el proceso de enseñanza; que el estudiante logre ser capaz de comprender, con herramientas filosóficas, la labor filosófica misma, encontrándole sentido. Para eso necesita admirarse y entender. Pero también necesita 
ser capaz de cuestionar e interpretar. Se interpreta cuando se entienden acciones en contextos diversos.

Como se ve, el proceso termina en su punto de partida. El que comprende no es el que ha llegado a todas las respuestas sino el que está capacitado para hacer más preguntas. Esto hace parte de la esencia de la actividad filosófica.

\section{La formación ética}

La enseñanza de la filosofía también puede servir para fortalecer disposiciones humanas relativas a los ámbitos ético y estético. Todo ser humano es un ser moral, y en ese sentido, toda buena propuesta educativa debe estar orientada al fortalecimiento tanto de las disposiciones de carácter o temperamento (o virtudes prácticas), como al fortalecimiento de las disposiciones racionales, emotivas e intuitivas de la acción moral correcta (las virtudes intelectuales y los sentimientos morales). En líneas generales, filosofar sirve para la felicidad, porque como guía teórica y práctica puede hacer que el estudiante se haga más consciente de su condición humana y ponga en práctica las enseñanzas que una razón práctica (o una sabiduría práctica o régimen de prudencia) le pueda ofrecer. No es un secreto que la filosofía aborda directamente los problemas del sentido de la vida, la consecución de la felicidad, la determinación de los ideales y valores, el fundamento de la norma moral, y la posibilidad de la libertad. A través del estudio de la filosofía, que no es otra cosa que la práctica de la reflexión filosófica, se puede aprender a ser feliz y a ejercer la libertad con responsabilidad. Específicamente, el estudio de la filosofía en bachillerato fortalece virtudes prácticas, que en principio pueden ser asumidas como disposiciones de carácter, pero que en vista de la necesidad de ser ejercitadas en la acción para generar buenos hábitos, pueden ser observadas como "competencias" éticas. Las definiciones propuestas de tales virtudes prácticas sólo están construidas, como es evidente, a partir del papel que juega la reflexión filosófica en su desarrollo:

- Honestidad: Virtud implícita en la misma búsqueda libre, desinteresada y consciente de la verdad, propia de la reflexión filosófica.

- Responsabilidad: Virtud inherente a cualquier actividad intelectual que se realice, ya sea con miras al conocimiento en sí mismo, como en función de su bondad práctica individual o social. La responsabilidad en la reflexión filosófica es el ejercicio consciente de la libertad de pensamiento. La búsqueda de la verdad, al ser difícil, es exigente. Implica tanto disciplina (orden, transparencia, constancia) como compromiso (deseo real reconocido como un deber). El individuo que filosofa debe dar cuenta de su filosofar; responde por lo que piensa. La vida académica, y en particular el debate filosófico, se conduce según una serie de reglas de acción habitualmente tácitas. Vale la pena hacerlas explícitas, a modo de ejemplo, en términos de deberes:

- Deber de hacerse entender y de ofrecer razones: el que filosofa tiene el deber de explicar y argumentar, lo mejor que pueda, lo que piensa en lo que dice. 
- Deber de comprender tesis distintas: el que filosofa tiene el deber de tener en cuenta lo que otros piensan, poniéndose en el lugar de los otros, tratando de entender, en el mejor sentido posible, lo que otros explican (principio de caridad), reconociendo la fortaleza o debilidad de los argumentos opuestos.

- Deber de responder a los cuestionamientos: el que filosofa tiene el deber de responder argumentadamente a las objeciones, preguntas, requerimientos, exigencias y argumentos de sus interlocutores.

Estos deberes a su vez implican, como es lógico, sus respectivos derechos.

- Prudencia (phrónesis) o sabiduría práctica: Virtud general relativa a la capacidad de reflexionar y sopesar con sensatez y solicitud tanto las decisiones y acciones particulares, como los proyectos de vida.

La filosofía, en un sentido ético fuerte, prepara para la vida en comunidad. Fortalece en los individuos las motivaciones y disposiciones necesarias para la vida política y para la construcción mancomunada de proyectos, en la medida en que hace consciente al individuo del ejercicio responsable de su libertad. Aunque suene extraño, el escepticismo, que es una forma particular de la filosofía entre otras, es un buen ejemplo. El escéptico filosófico por naturaleza es prudente y comedido, no actúa sin haber pensado muy bien antes los motivos, la naturaleza y el contexto de su acción, y puede colaborar, por tanto, en la construcción razonable de una idea de sociedad que constantemente hay que volver a pensar.

Aunque la práctica de la filosofía puede permitir una comprensión de los problemas políticos que ayuda a liberar a las personas, orientando la acción hacia la búsqueda de un bien común realizable, la lectura poco orientada de textos filosóficos puede sin embargo producir más sufrimiento del necesario y generar, en ocasiones, más vicios que virtudes. Mientras que la filosofía puede ayudar a formar personas más felices, la lectura desorientada de textos filosóficos puede ayudar a crear individuos arrogantes e infelices que, asociados con otros, se empeñen en lograr la infelicidad de los demás. La enseñanza de la filosofía tiene indudablemente un valor político, puesto que representa al mismo tiempo una esperanza para la felicidad común y una amenaza para la vida social. El encargado de dar clases de filosofía en el bachillerato tiene, por tanto, una responsabilidad enorme. Sobretodo porque la mayoría de sus estudiantes no van a consagrar su vida a la filosofía. Si el que enseña filosofía en bachillerato se empeña en enseñar a filosofar, subordinando sus gustos, preferencias o pasiones a este objetivo, muy probablemente logrará que la amenaza se convierta en esperanza y el vicio en virtud admirable. Pero si prefiere como objetivo inyectar gustos, preferencias o pasiones particulares propias en jóvenes fácilmente manipulables, confundiendo enseñar a pensar con enseñar a opinar como el profesor, dará muerte a la enseñanza de la filosofía y a la filosofía misma. El riesgo de la enseñanza de la filosofía en bachillerato no consiste solamente, por tanto, en dictar listados aburridos de compilaciones doxográficas que nadie entiende.

Las disposiciones emotivas e intuitivas que pueden ser condición de la conducta virtuosa son susceptibles de ser observadas bajo dos aspectos. En tanto que están relacionadas con las virtudes prácticas y las virtudes intelectuales pueden ser desarrolladas como si fuesen "competencias", pues son en buena medida tanto la fuente como el efecto del desarrollo 
de las "competencias" éticas. En palabras simples, el ejercicio de la virtud no sólo desarrolla el hábito de la virtud sino que también puede desarrollar la sensibilidad ética (de todo tipo, personal o social) al romper las barreras mentales que obstaculizan el despertar de tales sentimientos. Pero, en un segundo aspecto, y hablando con propiedad, las disposiciones emotivas e intuitivas no son competencias y constituye un riesgo el que sean evaluadas en la medida en que pueden ser falsamente visibles. Esto significa que el desarrollo de las competencias éticas debe tener en cuenta la posibilidad de estimular la sensibilidad ética pero no puede concentrarse ni reducirse a ello, en la medida en que no está en manos de la reflexión filosófica per se producir tales efectos emotivos, aunque sí pretenda generar reacciones prácticas (el ejercicio habituado de las virtudes éticas).

Desde un punto de vista estético, el estudio de la filosofía estimula también la sensibilidad artística. Ayuda a pulir, a perfilar y a hacer más exquisito nuestro sentido del gusto, dándole mayor sentido a las experiencias estéticas, favoreciendo el disfrute de lo bello y lo sublime, y ofreciéndole a la ética criterios más amplios de valoración y decisión moral fundados en una visión estética del mundo. Entre otras cosas, la filosofía se puede asumir como una manifestación estética o como un arte. En ella también hay la posibilidad del disfrute y la creación en sus más altas manifestaciones.

Evidentemente, para la actividad científica y académica la filosofía ofrece una buena motivación. El afán humano de entender y comprender está muy bien expresado en la búsqueda metódica de la verdad propia del filósofo y, detrás o a la par, de toda actividad científica existen, por supuesto, diversos modelos filosóficos. Pero la filosofía ofrece algo adicional, y es la posibilidad de dudar o cuestionarse con argumentos o razones los conocimientos ya obtenidos. La ciencia también hace esto, pero la filosofía lo asume de una manera distinta, a veces más radical. El ejercicio filosófico constituye un reto continuo para el dogmatismo, el extremismo, el fanatismo y el sectarismo. En ese sentido la filosofía es una buena terapia para aquellos que están muy convencidos de sí mismos, de lo que creen y practican, y no están por tanto dispuestos a aprender de sus errores. Es probable que a muchos -hay pruebas históricas de esto- la terapia filosófica les genere una reacción molesta, puesto que entrar en crisis, de suyo, no tiene por qué ser agradable. Pero no hay nada más temible que la ignorancia segura de sí misma, o las convicciones inamovibles que no se cuestionan por miedo a reconocer que tal vez se encuentran en el aire. La filosofía nos cura de esa trampa. Esa actitud prevenida ante el dogmatismo es inherente a las ciencias, pero a veces se nos olvida. La filosofía nos la recuerda.

Finalmente, se puede decir que la filosofía fortalece la fe. O mejor dicho, ayuda a hacerla honesta, definida y consciente. Dudar de sí mismo, cuestionarse, entrar en crisis, para luego encontrar sentido, fortalecerlo y asumirlo libre y felizmente, es algo que se puede encontrar en la práctica de la filosofía, y tales experiencias no están para nada alejadas de la experiencia religiosa. La conciencia o práctica de una fe, como experiencia natural humana, no entra necesariamente en contravía con el espíritu filosófico. La filosofía de suyo no destruye las formas religiosas de vida. En el contexto cultural colombiano, los entornos educativos comprometidos con el cristianismo, católico o no, o con el judaísmo o el Islam, no deben tenerle miedo a enseñar a filosofar. Identificar la filosofía con el 
laicismo, el ateísmo o el agnosticismo es confundir posturas filosóficas particulares con la reflexión filosófica como tal.

\section{Qué no es la filosofía y cómo enseñarla}

A pesar de todo lo dicho, creo que es necesario aclarar varias cosas. La filosofía no es un cuerpo doctrinal, ni un conjunto de teorías, ni una suma de ideas. No hay nada definitivo en filosofía, y por eso tal vez poco se la comprende. La filosofía es fundamentalmente problemática. Más que teorías filosóficas, o lo que popularmente se llama filosofías, lo que existen son caminos de reflexión filosófica. Así, si bien el estudio de la filosofía sirve para muchas cosas (y no creo que sólo las que en términos generales he mencionado) no es un saco de respuestas ni tiene por qué resolver de manera definitiva ninguna dificultad. Por esta razón generalmente la gente es reacia al estudio de la filosofía, le teme o lo desprecia. Esto es natural, pues parece ser que los seres humanos tendemos a buscar más seguridad que incertidumbre, queremos más lo estable y resuelto que lo dudoso y problemático. Pero este rechazo es también debido a una profunda incomprensión de la naturaleza de la actividad filosófica. Si se asume de entrada que la filosofía es más un método que un cuerpo de verdades, que es más una terapia para desprendernos de nuestros prejuicios infundados que un recetario de respuestas pragmáticas, que es en suma más de preguntas que de soluciones, es probable que ese rechazo (que generalmente se da por puro desconocimiento de la materia) desaparezca. Pero, para lograr eso se necesita explicar la necesidad de esas preguntas y métodos de reflexión llamados filosóficos.

La buena enseñanza de la filosofía debe procurar que el estudiante por sí mismo logre plantearse preguntas, vea que surgen naturalmente y comprenda su necesidad. Un buen programa de filosofía debe estar orientado en ese sentido, a que el estudiante se dé cuenta de que naturalmente se ve impelido a filosofar, y que esa disposición exige de ciertas competencias que se pueden desarrollar. En el proceso de enseñanza se espera que el estudiante se dé cuenta de que para ser feliz necesita filosofar de alguna forma. Si esto se logra podrá en definitiva comprender por qué ha existido la filosofía, por qué sigue existiendo, por qué es importante, por qué la ha estudiado, etc. Esta consciencia de la necesidad de la filosofía es indispensable para que el estudiante comprenda en concreto los caminos de reflexión filosófica y su historia. Pero al mismo tiempo, sólo es posible que el estudiante comprenda la necesidad de la filosofía y se sirva de ella para su vida si logra comprender en concreto cómo algunos filósofos de diversos tiempos han pensado las preguntas que al estudiante inquietan junto con preguntas que no tienen por qué inquietarle tanto pero a las que después de todo puede encontrarles su sentido. Puesto que la comprensión de la historia de los caminos de reflexión filosófica resulta indispensable para comprender de hecho la labor filosófica, un currículo debe también ofrecer una aproximación histórica a la actividad filosófica. Aproximación que, por supuesto, no puede quedarse quieta en una sola visión de la historia asumida acríticamente.

Todos sabemos que no se puede hacer filosofía sin método. Entrar crudamente a la reflexión filosófica de preguntas asombrosas puede ser en buena medida motivador, 
pero basta una breve incursión en el terreno para que el desaliento y la decepción aparezcan si no se poseen herramientas adecuadas de reflexión. Cuando se empieza a filosofar desprevenidamente, en vista de la dificultad de algunas preguntas asombrosas, la gente entra rápidamente en estados de confusión y de incertidumbre, empieza a generar un rechazo natural hacia ese tipo de reflexión y busca una salida dogmática pero práctica al embrollo. Todo responsable de la enseñanza de la filosofía en bachillerato debe estar prevenido ante esto, preparando previamente al estudiante para que tenga algunos instrumentos indispensables para enfrentar las dudas que el pensamiento filosófico plantea. Un buen currículo se inicia con la puesta en práctica de esas herramientas, que no son otras que las de la lógica y la argumentación, en contextos y sobre temas muy cercanos a los estudiantes. Esto asegura que el estudiante no se sienta aprisionado en los temas de reflexión filosófica y sepa que las dificultades tienen alguna salida. Al mismo tiempo le ayuda a comprender mucho mejor de qué se está hablando.

Algazel. Confesiones. Madrid: Alianza, 1989.

Aristóteles. Tratados de lógica. Madrid: Gredos, 1982. Ëtica Nicomáquea. Madrid: Gredos, 1993.

Averroes. Discours décisif. Paris: Flammarion, 1996.

Benjamin, A. Cornelius. “The Philosophy Instructor: Teacher or Preacher?”. En: Journal of Higher Education. Vol.33, No. 8, Nov 1962, pp.409-416.

Derrida, Jacques. La filosofía como institución. Barcelona: Juan Granica, 1984.

Díaz, Jorge Aurelio. "Una crítica romántica al romanticismo". En: Ideas y Valores. No.104, Ago 97, pp.29-36.

Fernandez Falagan, Pedro. "La ensenanza-aprendizaje de la filosofia. Bibliografia española complementaria". En: Diálogo Filosófico. Vol. 13, No. 39, Sep-Dic 1997, p. 319-333.

Gilson, Etienne. History of Philosophy and Philosophical Education. Aquinas Lecture 1947, Fall. Milwaukee: Marquette University, 1948.

Hinrichs, Gerard. "On the Teaching of Philosophy". Journal of Higher Education. Vol.23, No. 3, Mar 1952, pp.148-152.

Hoyos-Vasquez, Jaime. "El seminario en la experiencia docente en la Facultad de Filosofia de la Pontificia Universidad Javeriana”.En: Universitas Philosophica. Vol. 6, No. 10, Jun 1988, p. 39-53.

Lledó, Emilio. "La enseñanza de la Filosofia". En: Investigacion y Ciencia. No. 239, Ago 1996; p. 20-27.

Nietzsche, Friedrich. Sobre el porvenir de nuestras escuelas. Barcelona: Tusquets, 2000.

Perry, Charles M. "The Place of Philosophy in Universities". En: Journal of Higher Education. Vol.13, No. 9, Dic 1942, pp.463-470.

Russell, Bertrand. "Educación”. En: Escritos básicos II. Barcelona: Planeta-De-Agostini, 1984.

Salazar Paniagua, Freddy. "La historia de la filosofia en la enseñanza de la filosofia". En: Revista de la Universidad Industrial de Santander. Humanidades Vol. 28, No. 1, Ene.-Jun 1999, p. 41-48.

Schopenhauer, Arthur. Sobre la filosofía de universidad. Madrid: Tecnos, 2001.

Thomas, J.L.H. "The Schoolman's Advocate: in Defense of the Academic Pursuit of Philosophy". En: Mind. New series, Vol.28, No. 392, Oct 1989, pp.483-506.

Recibido 18/09/07. Aprobado 19/10/07 